\title{
Proposed laboratory surrogate markers for the management of acute kidney injury in ethylene glycol poisoning in resource-challenged countries; a Mayo Clinic Health System, Wisconsin, case analysis
}

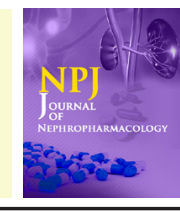

\author{
Macaulay Amechi Onuigbo ${ }^{1,2}$, Eileen Samuel ${ }^{2}$, Nneoma Agbasi ${ }^{3}$, Adel Zurob ${ }^{4}$ \\ ${ }^{1}$ Mayo Clinic College of Medicine, Rochester, MN, USA \\ ${ }^{2}$ Department of Nephrology, Mayo Clinic Health System, Eau Claire, WI, USA \\ ${ }^{3}$ North East London NHS Foundation Trust, United Kingdom \\ ${ }^{4}$ Department of Pulmonary Medicine, Mayo Clinic Health System, Eau Claire, WI, USA
}

\section{A R T I C L E I N F O}

Article Type:

Case Report Analysis

\section{Article History:}

Received: 11 October 2017

Accepted: 15 December 2017

ePublished: 5 January 2018

\section{Keywords:}

Acute kidney injury

Ethylene glycol

Hemodialysis

Laboratory surrogate markers

Renal replacement therapy

Resource-poor countries

\begin{abstract}
A B S T R A C T
Mass poisonings with diethylene glycol (DEG) usually ingested as an unintended component of pharmaceutical preparations have occurred in Nigeria, especially affecting children with nearly $100 \%$ mortality outcomes. Clearly, the unavailability of Specialty Toxicology laboratory testing facilities translates to non-diagnosis and therefore inappropriate management of these patients with resulting poor outcomes. In this case analysis, while utilizing a case report involving intentional DEG intoxication that we managed at the Mayo Clinic Health System in North-Western Wisconsin, we have demonstrated the utility of serial values of commonly available blood test parameters including serum bicarbonate and anion gap, blood ABG pH and $\mathrm{CO}_{2}$ values, together with the use of serum osmolality gap as surrogates to expedite early and appropriate treatment of such cases even in the absence of Toxicology confirmation of $\mathrm{DEG}$ as the poisoning agent. Based on the experience gained from this case report, we strongly recommend that the responsible governments in resource-challenged countries must ensure that these laboratory surrogate indices, at the minimum, become available in the hospitals. This would improve outcomes in general.
\end{abstract}

Implication for health policy/practice/research/medical education:

Over the last several decades, mass poisonings of diethylene glycol (DEG), usually ingested as an unintended component of pharmaceutical preparations, have occurred in Nigeria, especially affecting children with nearly $100 \%$ mortality outcomes. The unavailability of toxicology laboratory testing facilities often translates to non-diagnosis and therefore inappropriate management of these patients. In this case analysis, we propose the utility and monitoring of serial values of commonly available blood test parameters such as serum bicarbonate and anion gap, blood $\mathrm{ABG}$ pH and $\mathrm{CO}_{2}$ values, together with the use of serum osmolality gap to expedite early and appropriate treatment of such cases. Moreover, it remains the obligate duty of the various governments in these countries to ensure the availability of requisitely necessary ancillary laboratory testing services in their areas of jurisdiction.

Please cite this paper as: Onuigbo MA, Samuel E, Agbasi N, Zurob A. Proposed laboratory surrogate markers for the management of acute kidney injury in ethylene glycol poisoning in resource-challenged countries; a Mayo Clinic Health System, Wisconsin, case analysis. J Nephropharmacol. 2018;7(1):46-49. DOI: 10.15171/npj.2018.11.

\section{Introduction}

On November 18, 2008, the Nigerian Federal Ministry of Health $(\mathrm{FMOH})$ received a report of 13 cases of unexplained acute renal failure (ARF), or acute kidney injury (AKI), among children from a hospital in Lagos state. Several of the patients had been exposed to a liquid acetaminophen-based teething medication. On November
21, officials from the Nigerian National Agency for Food and Drug Administration and Control (NAFDAC) discovered diethylene glycol (DEG) in four batches of the teething medication manufactured during AugustOctober 2009 (1). In 2008-2009, there was a spate of fatal accidental poisonings associated with unexplained ARF among Nigerian children following the use of medications 
contaminated with ethylene glycol (1). Fifty-four of 57 (95\%) children investigated died (1). Over the last several decades, mass poisonings of DEG, usually ingested as an unintended component of pharmaceutical preparations, have occurred (2). In order to promptly halt the rise in deaths due to ingestion of these pharmaceuticals, laboratory analysis has often been employed to identify and quantify the etiologic agent after the medications have been tentatively implicated (2). Over the past 15 years, the Centers for Disease Control and Prevention has been involved in identifying DEG in implicated pharmaceutical products during three poisoning epidemics that occurred in Nigeria (1990), Haiti (1995), and, most recently, in Panama (2006) (2). An earlier 1992 report from Nigeria revealed that between June and September 1990, 47 children died at Jos University Teaching Hospital, Nigeria from ingestion of paracetamol syrup adulterated with DEG (3). Most of the children presented with anuria, fever, vomiting, diarrhea and convulsions (3). Signs on admission were tachycardia, acidotic breathing, pallor, oedema and hepatomegaly (3). Laboratory findings included hyperkalaemia, acidosis, elevated creatinine level and hypoglycaemia (3). Management, as documented in this report consisted of correction of dehydration and acidosis plus administration of antibiotics when indicated (3). All (100\%) died within 2 weeks of admission and, notably, we have observed, none of the children received dialysis (3).

We posit here, that very clearly, the timely identification of such intoxications would have resulted in much better patient outcomes. However, as was evident in similar reports from resource-challenged countries, there is a paucity of toxicology laboratory services to identify the culprit intoxicants (1-3). We propose that the utility of laboratory surrogates that are more commonly available in resource-challenged countries in such clinical scenarios could advance the quality of care provided in such settings with improvements in critical life-saving and renal-salvage outcomes.

\section{Results of case report analysis}

In October 2014, at the Mayo Clinic Health System in Northwestern Wisconsin, USA, our Nephrology Service had managed a 28-year old Caucasian male with a past history for major depressive disorder and paranoid personality features and cluster B personality features following observed brake fluid ingestion as a suicidal intent. The patient had earlier presented himself to the registration desk in the Emergency Department, stating that he needed "detox" and shortly after that, he began to shake and soon was unresponsive. An observer had reported to the medical staff in the Emergency Department that she had observed the patient drinking a bottle of "something" in the parking lot prior to coming in, and the empty bottle of brake fluid was confirmed at the site subsequently. The patient had also intentionally swallowed several $1 \mathrm{mg}$ clonazepam tablets.

He was immediately intubated for unresponsiveness in the emergency department and transferred to the critical care unit (CCU). He was unresponsive, hypotensive and intubated and intravenous norepinephrine was quickly initiated for blood pressure support. His initial ABG pH of 7.33 at 1412 hours had rapidly deteriorated to a value of 7.08 at 1605 hours, less than two hours after his admission to the hospital. Toxicology samples were immediately sent to an out-of-state toxicology laboratory which subsequently confirmed ethylene glycol intoxication.

$\mathrm{He}$ was resuscitated with intravenous fluid infusions and emergently received intravenous fomepizole for detoxification. Emergent hemodialysis was started with rapid improvement in patient's physiology. He required daily prolonged hemodialysis treatments during the first three days of the admission.

We had methodically monitored the trajectories of the following relatively easily measurable laboratory indices during the initial phase of his hospital stay in the CCU; basic metabolic profile, $\mathrm{ABG} \mathrm{pH}, \mathrm{ABG} \mathrm{CO}_{2}$, serum bicarbonate, serum anion gap, measured serum osmolality and serum osmolal gap. Additional complications during this hospitalization included acute respiratory failure with aspiration pneumonia, coagulopathy with platelet reduction, and anemia. He recovered after several days in the CCU and was then transferred to the behavioral health unit. He was discharged from the hospital 24 days later. The trajectories of concurrent serum bicarbonate, blood $\mathrm{ABG} \mathrm{CO}$, blood $\mathrm{ABG} \mathrm{pH}$, anion gap, and serum osmolality values in our patient are shown below in Figures 1-5.

A very meticulous analysis of these trajectories would confirm that the monitoring of almost any one of these single agents could have been an appropriate guide to the patient's management, even without recourse to any toxicology laboratory data. An ABG pH value of 7.08 with an $\mathrm{ABG} \mathrm{CO}$ of $14 \mathrm{mmol} / \mathrm{L}$ and an anion gap of 27 clearly translated to a diagnosis of severe high anion gap metabolic acidosis. These observations, in the setting of rising serum creatinine (Figure 6), i.e. AKI, should have triggered an

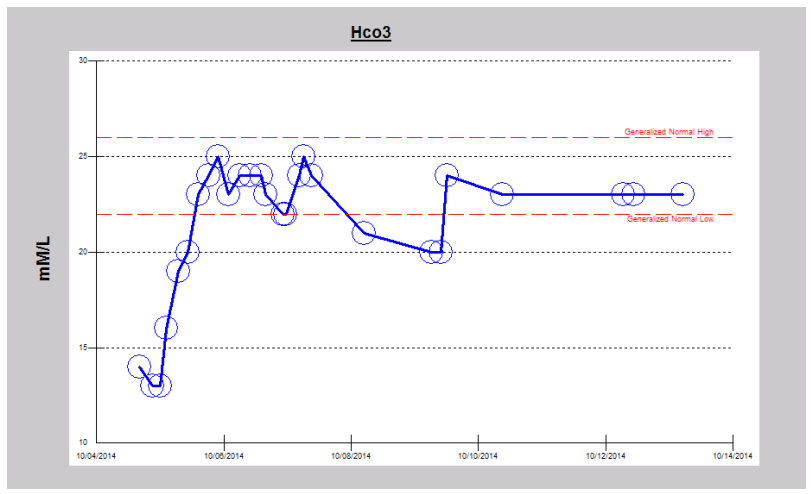

Figure 1. Trajectory of serum bicarbonate during the first 10 days of the admission with intentional ethylene glycol intoxication. 


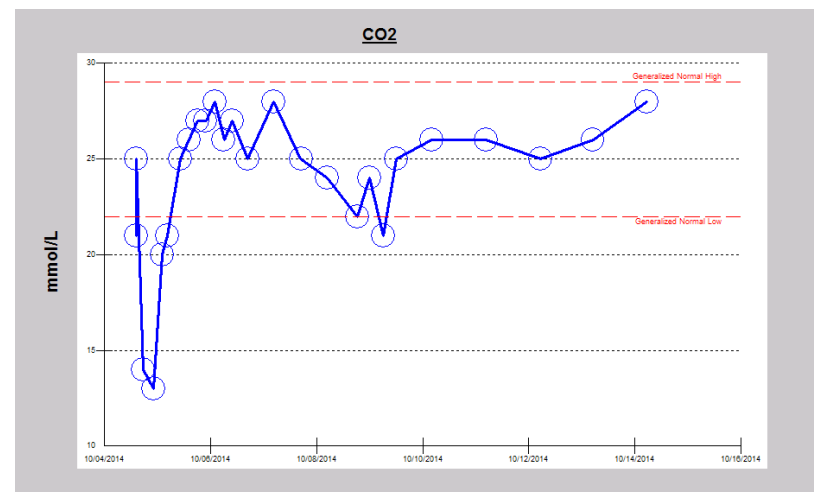

Figure 2. Trajectory of blood ABG $\mathrm{CO} 2$ during the first 10 days of the admission with intentional ethylene glycol intoxication.

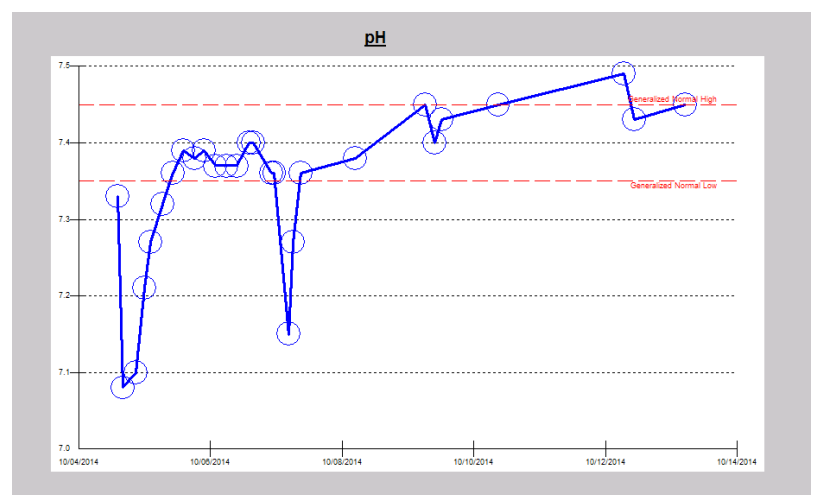

Figure 3. Trajectory of blood $A B G \mathrm{pH}$ during the first 10 days of the admission with intentional ethylene glycol intoxication.

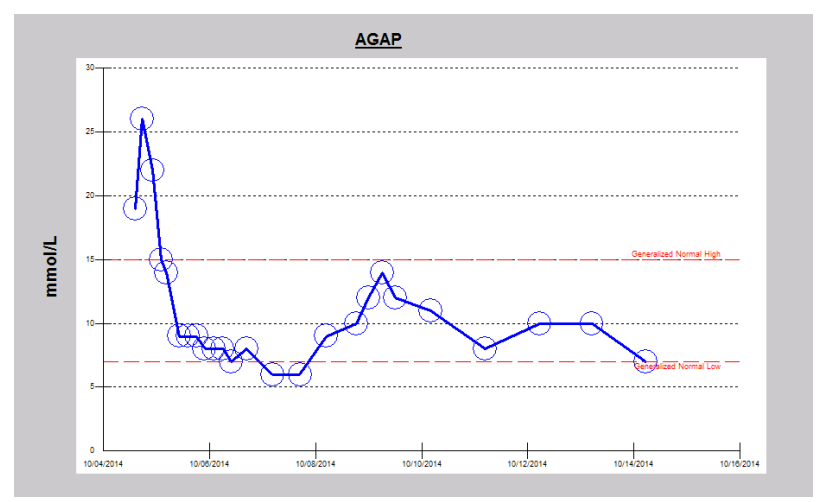

Figure 4. Trajectory of serum anion gap during the first 10 days of the admission with intentional ethylene glycol intoxication.

indication to consider renal replacement therapy, even in a resource-poor environment. Where renal replacement therapy (dialysis) services are not available, then an urgent transfer would have been mandatory. Furthermore, subsequent monitoring of such laboratory surrogate markers as serum bicarbonate, anion gap, serum osmolal gap (measured minus calculated serum osmolality; Figure 5 ), with or without the $\mathrm{ABG}$ parameters of $\mathrm{pH}$ and $\mathrm{CO}_{2}$, would easily have enabled the offering of an excellent care to such patients.

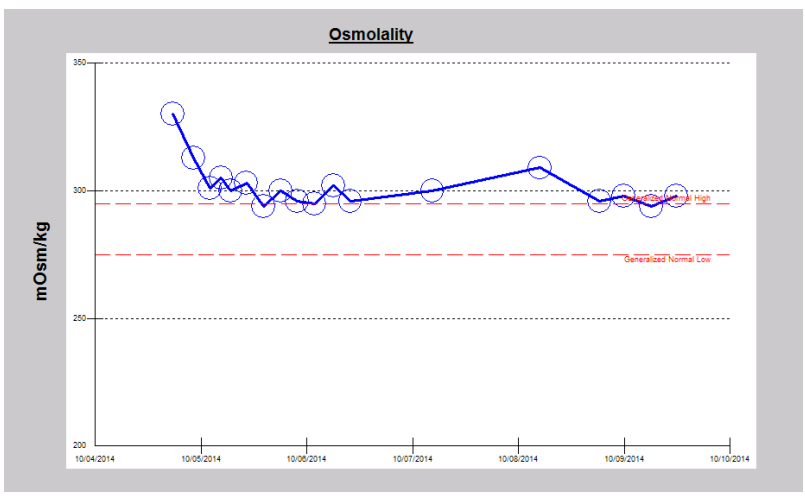

Figure 5. Trajectory of measured serum osmolality during the first 10 days of the admission with intentional ethylene glycol intoxication.

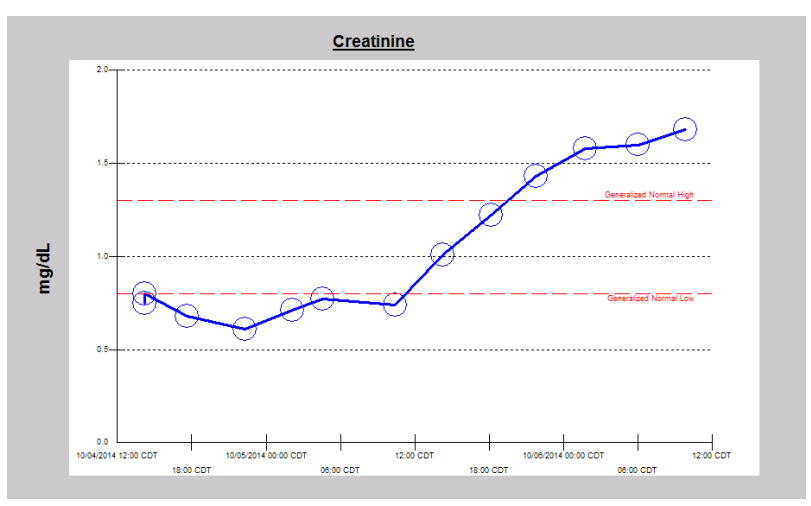

Figure 6. Trajectory of rising serum creatinine during the first 48 hours of the admission with intentional ethylene glycol intoxication.

\section{Discussion}

In a related publication, Akuse et al from the Department of Pediatrics, Ahmadu Bello University Teaching Hospital, Zaria, Nigeria, had in 2012, in a multicenter study, investigated whether AKI associated with DEG could be identified by other means (4). Compared to children who had not ingested DEG, children with DEG poisoning were significantly $(P<0.05)$ younger $(11.95 \%$ vs. 31 months), more were anuric (98.3\% vs. $74.6 \%)$, hypertensive ( $84 \%$ vs. $52 \%$ ), had severe metabolic acidosis ( $46.7 \%$ vs. $20.5 \%)$, and died ( $96.6 \%$ vs. $71.2 \%$ ) (4). They had developed increasing metabolic acidosis and multiorgan dysfunction despite peritoneal dialysis (4). Late presentation, financial difficulties, inadequate facilities for toxicology, and hemodialysis complicated management (4).

The analysis of the trajectories of the above indices in our case report has demonstrated the usefulness and utility of these more easily available laboratory indices as surrogates for DEG. The attending physician could therefore institute prompt and appropriate treatment modalities very early, even when the specific identification of the culprit intoxicant may never be possible in resource-challenged settings (1-4). This would result in much improved patient 
outcomes in many of these cases of unexplained AKI.

Based on the experience gained from this case report, we strongly recommend that the responsible governments in resource-challenged countries must ensure that these surrogate indices, at the minimum, are available in the hospitals. This would improve outcomes in the management of unexplained AKI. Furthermore, at least in the pediatric population, based on the experience of Akuse et al, it would appear that hemodialysis would be a better renal replacement modality than peritoneal dialysis (4).

\section{Conclusion}

We finally posit that the lack of adequate diagnostic facilities may explain the paucity of reports of such intoxications and poisonings in Nigeria in particular, and in other resource-poor countries, in general. It is likely that we have been missing them as they were never identified as cases of say DEG intoxication.

\section{Authors' contribution}

MAO: Conception, design, acquisition of data, data analysis, interpretation of data, literature review, drafting the article and final approval of manuscript. ES: Acquisition of data, literature review and final approval of manuscript. NA and AZ: Critical revising for important intellectual content, design, and final approval of manuscript.

\section{Conflicts of interest}

The authors report no conflicts of interest. The authors alone are responsible for the content and writing of the article.

\section{Ethical considerations}

Ethical issues (including plagiarism, data fabrication, double publication) have been completely observed by the authors. The patient has given his informed consent $t 0$ publish this case report.

Funding/Support

None.

\section{References}

1. Centers for Disease Control and Prevention (CDC). Fatal poisoning among young children from diethylene glycol-contaminated acetaminophen Nigeria, 2008-2009. MMWR Morb Mortal Wkly Rep. 2009;58:1345-7.

2. Barr DB, Barr JR, Weerasekera G, Wamsley J, Kalb SR, Sjödin A, et al. Identification and quantification of diethylene glycol in pharmaceuticals implicated in poisoning epidemics: an historical laboratory perspective. J Anal Toxicol. 2007;31:295-303.

3. Okuonghae HO, Ighogboja IS, Lawson JO, Nwana EJ. Diethylene glycol poisoning in Nigerian children. Ann Trop Paediatr. 1992;12:235-8.

4. Akuse RM, Eke FU, Ademola AD, Fajolu IB, Gbelee $\mathrm{HO}$, Ihejiahi $U$, et al. Diagnosing renal failure due to diethylene glycol in children in a resourceconstrained setting. Pediatr Nephrol. 2012;27:1021-8. doi: $\quad 10.1007 / \mathrm{s} 00467-011-2082-8$.

Copyright $\odot 2018$ The Author(s); Published by Society of Diabetic Nephropathy Prevention. This is an open-access article distributed under the terms of the Creative Commons Attribution License (http://creativecommons.org/licenses/by/4.0), which permits unrestricted use, distribution, and reproduction in any medium, provided the original work is properly cited. 